\title{
Bioscientific topics concerning Aspergillus oryzae, the national microorganism of Japan.
}

\author{
Eiji Ichishima ${ }^{1,2 *}$ \\ ${ }^{1}$ Department of Applied Biological Chemistry, Tohoku University, Sendai, Japan \\ ${ }^{2}$ Department of Agricultural Chemistry, Tokyo University of Agriculture and Technology, Tokyo, Japan
}

\begin{abstract}
Traditional Japanese cuisine ("Washyoku" in Japanese) was added to the UNESCO's Intangible Culture Heritage list in 2013. Over the past 1,000 years, the use of fungal hydrolytic enzymes prevailed in the Japanese fermentation

industries. Almost all characteristic flavors and tastes of these traditional fermented foods are developed using the malted rice with Aspergillus oryzae ("kōjip" in Japanese). In 2006, the Brewing Society of Japan certified A. oryzae, A. sojae and A. Iuchuensis as the national microorganisms of Japan.

In this review, a novel enzymatic production of glutamate from glutamine and new Skn-1a/Pou2f3 umami taste receptors are discussed. Biological characteristics for Aspergillus, such as the AoSO protein accumulation at the septal pore, and the large non-conserved region of the tethering protein Leashin, are presented and described here, together with the analyses of $\alpha$-amylase, ManR functions, S21 family glycine-D-alanine aminopeptidase (GdaA), and ionic interactions between the fungal hydrophobin RolA and cutinase CutL1. Furthermore, I discuss the development of antihypertensive peptide (GY, SY)-enriched soy-sauce-like seasoning and the bifidogenic effect of supplemental acid protease from $A$. oryzae.
\end{abstract}

Keywords: Aspergillus oryzae, Aspergillus sojae, Glutamic acid, kōji, National microorganism, Umami.

Accepted on 5 January, 2018

\section{Introduction}

Over the past 1,000 years, the use of hydrolytic enzymes from fungi has become the prevalent Japanese fermentation method for saké brewing when making the offerings in the Shintoshrines [1-4]. Generally, in Japan, alcoholic drinks have been produced primarily using rice $[2,4,5]$.

The role of $k o j j i$ during saké brewing is to supply the enzymes, which break down rice starch to glucose during the mashing processes, and vitamins, amino acids and other molecules necessary for yeast growth. Additionally, $k \overline{o j i}$ is a useful additional glucose source due to the starch it contains [5]. To obtain $k \overline{o j i}$, seed $k \overline{o j i}$ (Tané $k \overline{o j i}$ or Moyashi in Japanese) is obtained from Aspergillus oryzae strains. It has been used throughout Japanese history, and in the cooking and serving techniques dating over 1,000 years ago. In Japan, many materials, such as soy sauce, Miso, Mirin, and rice vinegar, are used for growing A. oryzae. Additionally, a book containing recipes for healthy dishes using $k \overline{o j} i$ was recently published [6].

$A$. oryzae is generally recognized as a non-pathogenic fungus. Safty and non-productivity of aflatoxin have been well established in the industrial strains [7], and this fungus is considered "generally recognized as safe (GRAS)" by the United States Food and Drug Administration (FDA) [8-10].

Molecular analysis of an inactive aflatoxin biosynthesis gene cluster in $A$. oryzae was reported by Tominaga et al. [11]. The genome of this fungus was sequenced in 2005 [12], while the genome of Aspergillus sojae [13] was sequenced in 2011 [14]. Since Inui $[15,16]$ identified Aspergillus luchuensis in black $k \overline{o j i}$ from Okinawa in 1901, many fungi associated with $k \overline{o j} i$ molds were reported as well [14,18,19,53]. Furthermore, the catalytic and molecular properties of unique enzymes obtained from Aspergillus strains used in Japanese bioindustries were analyzed [20,21], together with the development of enzyme technologies using $A$. oryzae, A. sojae, and A. luchuensis enzymes, the national microorganisms of Japan [22].

A. oryzae, was selected to represent the national microorganism of Japan during the meetings commemorating 100 years from the establishment of the Brewing Society of Japan in October 2006 [23,24]. In this review, bioscientific topics associated with $A$. oryzae and $A$. sojae, the national microorganisms of Japan, are presented and discussed.

\section{Renewed Interest for the Umami Taste}

\section{Glutamate}

In 1932, Udo [52] identified L-glutamate (monosodium glutamate) as the most important taste and flavor component of the Japanese traditional fermented food, soy sauce. During soy sauce fermentation, L-glutamate is produced through two pathways, including (i) the cleaving of proteins by proteases and/or peptidases and (ii) the hydrolysis of free L-glutamine obtained by protein cleavage by glutaminase (EC 3.5.1.2). The 
latter process is more important for enhancing umami taste, because L-glutamine can be converted to L-glutamate.

Ten genes encoding proteins with glutaminase activity were identified in the $A$. sojae genome; and one of them, GahB, a peptidoglutaminase-asparaginase, was shown to act as the main glutaminase in the soy-sauce kojji [46], which led to its purification from the cytoplasm of GahB-overexpressing strains (AsGahB). They also found that 4 of the 10 glutaminases, GahA, GahB, GatA, and Gls, were involved in glutamate production in soy sauce and that important for enhancing glutamate production [25]. A. sojae GahA (AsGahA) was purified from the cell surface in submerged culture as a complex of 3 polypeptides [26], polypeptides A, B, and C. Polypeptides B and C were partially cleaved products from the larger polypeptide A, and the secreted AsGahA was present as a cleaved form (polypeptide B) in solid-state culture [26]. Hence, proteolytic digestion might be involved in the localization of AsGahA and AsGhaB. In 2014, Ito and Koyama [27] demonstrated that a specific proteolytic digestion of as AsGahB by extracellular proteases of $k \overline{o j i}$ molds is similar to that of AsGahA, which exists in the proteolytic form in the solid-state cultures.

\section{Umami taste receptor}

Matsumoto et al. [28] identified Skn-1a/Pou2f3 as a specific taste receptor in a study published in Nature Neuroscience. Functional diversification of taste cells is crucial for proper discrimination of taste qualities, and these researchers demonstrated that homeodomain protein Skn-1a/Pou2f3 is expressed by sweet, umami, and bitter taste cells.

Taste signals and nutrient stimuli sensed by the components of gastrointestinal tract are transmitted to the brain, in order to regulate feeding behavior and energy homeostasis, which is referred to as the gut-brain axis. Ushiama et al. [29] proposed that the taste-sensing cells in the oral cavity and/or foodborne chemical-sensing brush cells in the gut are involved in the regulation of body weight and adiposity mediated by brain signaling. The discovery of food-derived factors that regulate the activity of these cells may offer new strategies for the treatment of obesity and diabetes

\section{Biological Characteristics of $A$. oryzae}

\section{AoSO protein accumulates at the septal pore of $A$. oryzae}

The septal pore of $A$. oryzae may play an important role in the growth of multicellular organisms. Maruyama et al. [30] identified an $A$. oryzae protein, AoSO, homologous to the Neurospora crassa SO protein, which was reported to accumulate at the septal pore in aging hyphae. The $\Delta$ Aoso strain showed excessive cytoplasmic leakage upon hyphal injury, similar to that observed in the Woronin body-deficient $\triangle$ Aohex1 strain. Furthermore, AoSO was shown to accumulate at the septal pore in response to various stresses, including low and high temperature, extreme acidic and alkaline $\mathrm{pH}$, and nitrogen and carbon depletion.

\section{The tethering protein Leashin in A.oryzae}

The Woronin body is a Perizomycotina-specific organelle that is typically tethered the septum, but upon hyphal wounding, it prevents excessive cytoplasmic loss by plugging the septal pore. Leashin (LAH) is a large Woronin body-tethering protein that contains highly conserved $\mathrm{N}$ - and C-terminal regions and a long ( 2,500 amino-acid) non-conserved middle region. As the involvement of the non-conserved region in Woronin body function has not been investigated [30].

Han et al. [31] characterized individual regions of Leashin protein of $A$. oryzae (AoLAH). In an Aolah disruptant, no Woronin bodies were tethered to the septum and hyphae had a reduced ability to prevent excessive cytoplasmic loss upon hyphal wounding. The N-terminal region of AoLAH was demonstrated to associate with Woronin bodies, through a process dependent on another molecule, Woronin body sorting complex of $A$. oryzae (AoWSC), a homologue of $N$. crassa WSC, while the C-terminal region of this protein was shown to localize to the septum. Using AoLAH N-terminal-regionenhanced green fluorescent protein (EGFP) fusion protein, elastic movements of Woronin bodies were observed. An Nand C-terminal fusion construct lacking the non-conserved middle region of AoLAH was observed to be sufficient for the tethering of Woronin bodies to the speptum, but Woronin bodies were located closer to the septum and the elastic movements were impaired. Moreover, the expression of middle-region-deleted AoLAH in the Aolah disruptant strain did not restore the ability of this molecule to prevent excessive cytoplasmic loss. Taken together, these results indicate that the non-conserved middle region of AoLAH is important for the regulation of the position, movement, and functioning of Woronin bodied.

\section{Potential cell wall component of $\alpha$-amylase in $A$. oryzae}

At later stages of cultivation of $A$. oryzae, a decrease in $\alpha$ amylase (Taka-amylase A; TAA) (EC 3.2.1.1) activity caused by adsorption of TAA onto the cell wall and its degradation by extracellular proteolytic enzymes [32]. Consequently, alkaliinsoluble cell wall fractions exhibited high levels of TAA adsorption, together with an increase in the adsorption capacity following the treatment with $\beta$-glucanase, which resulted in the concomitant increase in the amount of detected chitin. In contrast, the adsorption capacity was diminished by treating the cell wall fraction with chitinase (EC 3.2.1.14), suggesting that the cell wall component that allows TAA adsorption is chitin. However, the TAA adsorption was not possible on both the mycelium and cell wall at the early stages of cultivation, although the chitin content was not altered.

\section{ManR controls the $\beta$-mannan system utilization in $A$. oryzae}

Fungal endo $\beta$-mannanases ( $\beta$-mannanases, mannan endo-1,4mannnosidase, EC 3.2.1.78) are widely used as industrial enzymes. However, no transcriptional regulator of $\beta$ mannanases has been identified in fungi or other eukaryotic 
Citation: Eiji Ichishima. Bioscientific topics concerning Aspergillus oryzae, the national microorganism of Japan. J Biochem Biotech. 2018;1(1):42-46.

cells to date. ManR, a Zn (II) 2Cys6 type type DNA binding protein, was identified in A. oryzae [33]. ManR positively regulates the expression of not only $\beta$-mannanase $(\beta$ mannosidase, EC 3.2.1.25), but also the enzymes involved in the degradation of galactomannans and glucomannans such as $\alpha$-galactosidase (EC 3.2.1.22), $\beta$-mannosidase (EC 3.2.1.25), acetylmannan esterase, and $\beta$-glucosidase (EC 3.2.1.21). Ogawa et al. [34] reported that ManR controls the cellulose utilization system in $A$. oryzae.

\section{S21 family glycine-D-alanine aminopeptidase of $A$. oryzae}

The glycine-D-alanine aminopeptidase gene (gdaA) encoding a S21-family glycine-D-alanine aminopeptidase (GdaA) was identified in A. oryzae [35], and was shown to share $43 \%$ amino-acid sequence identity with the D-aminopeptidase (EC 3.4.11.19) of the gram-negative bacterium Ochrobactrum anthropi. Glycine-D-alanine aminopeptidase (GdaA) purified from an $A$. oryzae gdaA-overexpressing strain exhibited high $\mathrm{D}$-stereospecificity and efficiently released $\mathrm{N}$-terminal glycine and D-alanine at the optimal $\mathrm{pH}$ of 8 to 9 and at $40^{\circ} \mathrm{C}$. The enzyme was stable at $\mathrm{pH} 8$ to 11 and relatively resistant to acidic conditions up to $\mathrm{pH} 5$.

\section{Ionic interactions between fungal hydrophobin RolA and cutinase CutL1}

Hydrophobins are amphipathic proteins secreted by filamentous fungi [36]. In a liquid medium containing the polyester polybutylene succinate co-adipate (PBSA), A. oryzae produces a hydrophobin RolA, and cutinase CutL1, a PBSAdegrading cutinase (EC 3.1.1.74) [37]. Secreted hydrophobin RolA attaches to the surface of the PBSA particles and recruits cutinase CutL1, which then condenses on the particles and stimulates the hydrolysis of PBSA. Takahashi et al. [38] identified amino acid residues that are required for the hydrophobin RolA-cutinase CutL1 interactions by using sitedirected mutagenesis and quantitatively analyzed kinetic profiles of these interactions by using quartz crystal microbalance (QCM) technique. The QCM analyses revealed that Asp142. Asp171 and Glu31, located on the hydrophilic molecular surface of cutinase CutL1, and His32 and Lys34, located at the N-terminus of hydrophobin RolA, are crucial for the RolA-CutL1 ionic interactions.

\section{Antihypertensive effect of peptides-enriched soy- sauce-like seasoning}

Nakahara et al. [47] developed peptide-enriched soy sauce-like seasoning, named fermented soybean seasoning (FSS), by modifying the soy sauce brewing process. The angiotensinconverting enzyme (ACE, renin, EC 3.4.23.15) inhibitory activity of fermented soybean seasoning (FSS) (IC50 $=450$ $\mu \mathrm{g} / \mathrm{ml}$ ) was greater than that of regular soy sauce (IC50 $=1620$ $\mu \mathrm{g} / \mathrm{ml}$ ). The fermented soybean seasoning (FSS)-associated angiotensin-converting enzyme (ACE, renin) inhibition was shown to have antihypertensive effects on both spontaneously hypertensive rats and salt-sensitive (Dahl S) rats. By the way, salt-sensitive (Dahl S) rats exhibit many phenotypic rats associated with salt-sensitive hypertension in men.

The angiotensin-converting enzyme (ACE) inhibitory substances were purified from FSS by reverse phase chromatography and Ala-Trp (IC50=10 $\mu \mathrm{g} / \mathrm{mL})$, Gly-Trp (30 $\mu \mathrm{g} / \mathrm{mL})$, Ala-Tyr $(48 \mu \mathrm{g} / \mathrm{mL})$, Ser-Tyr $(67 \mu \mathrm{g} / \mathrm{mL})$, Ala-Phe $(690 \mu \mathrm{g} / \mathrm{mL})$, Val-Ile $(690 \mu \mathrm{g} / \mathrm{mL})$, Val-Gly $(1100 \mu \mathrm{g} / \mathrm{mL})$, and a nonprotein amino acid, nicotinamide $(0.26 \mu \mathrm{g} / \mathrm{mL})$, were identified as the substances responsible for the observed effects. Their concentrations in the FSS were shown to be higher than those in the regular soy sauce, Gly-Tyr (GY) and Ser-Tyr (SY) were identified as two primary substances in FSS contributing to the antihypertensive effects, by using spontaneously hypertensive rat (SMR) [48] and elucidated the effects of temperature on the stability of various peptidases during peptide-enriched soy sauce fermentation [39].

\section{Bifidogenic effect of an acid protease from A. oryzae}

Consumption of an acid protease derived from A. oryzae was shown to have bifidogenic effects in rats, with a considerable increase in Bifidobacterium content found in the cecum of rats fed with a high-fat diet supplemented with the $A$. oryzaederived Amano protease preparation [17]. The effect cannot be explained by the activity of acid protease at the concentration of $1-\mathrm{g} / \mathrm{kg}$ of this preparation, but four-fold higher concentrations of acid protease, compared with those initially used in the diet, were found to cause a significant bifidogenic effect.

Previously, aspartic acid was reported to be released following the trypsinogen activation by the trypsinogen-kinase found in $A$. oryzae $[49,50]$, which was shown to be identical to the $A$. oryzae-derived acid-protease [51]

In human duodenum, trypsinogen is converted to its active form, trypsin (EC 3.4.21.4), by membrane bound enteropeptidase (EC 3.4.21.9). Free trypsin then catalyzes the conversion of additional trypsinogen to trypsin and activates chmotrypsinogen, pro-carboxypeptidases, and proelastase. Acid protease (aspergillopepsin I, EC 3.4.23.18) from $A$. tubingensis (formerly designated as Aspergillus saitoi) [40] activates the generation of trypsin from trypsinogen [41], which was shown to be associated with the cleavage of the Lys6-Ile bond and the release of hexapeptide [42]. Previously, Asp76 of the acid protease, aspergillopepsin I, was identified as a binding site of the basic substrate, trypsinogen $[43,44]$.

In addition to these aspartic proteinases, trypsinogen is activated by $A$. oryzae-derived aorsin treatment as well [45] at $\mathrm{pH} 5.5$, and the generated trypsin hydrolyzes benzyloxycarbonyl-Phe-Arg-4-methylcoumaryl-7-amide (ZPhe-Arg-MCA) at pH 7.7, which is not cleaved by aorsin. IleVal-Gly-Gly-Tyr- was identified as the N-terminal sequence of the activated trypsin, indicating the accurate aorsin-mediated processing. It can be assumed that the A. oryzae-derived protease-activated trypsin further activates other inactive zymogens in a cascade reaction and that the bifidogenic effects of $A$. oryzae-derived acid protease increase with trypsin activation levels. 


\section{Conclusion}

In conclusion, the use of $A$. oryzae, the national microorganism of Japan, has a long and rich history. Furthermore, future studies of bioscientific topics concerning A. oryzae, presented in the review, may be very important for various processes and can be used to many beneficial ends in Japan and world.

\section{Acknowledgements}

I would like to dedicate this review to my coworkers, for their kind collaboration. This review was compiled to commemorate of our long research efforts, and I hope that the published results on Aspergillus oryzae will help other researchers in their studies.

\section{References}

1. Sakamoto T, Ienaga S, Inoue M. Nihon Shyo-ki. Part 1. Iwanami Shoten. 1993.

2. Ueda S. Origin of Japanese saké. (Nihonsyu No Kigen. Kabi, Kouji, Sake-No-Keifu). Yasaka-Syobou. 1999.

3. Yuzuki M. History of sake-brewing (Sake-Tukuri No Rekisi). Yū-zan-Kaku. 2003.

4. Sakaguchi K. The Japanese saké ("Nihon No Saké"). Iwanami Book Press. 2007.

5. Akiyama H. Textbook of saké brewing. The Brewing Society of Japan, Tokyo. 2016.

6. Onomisa M. Recipe book for healthy dishes using Kōji. ("Kōji No Recipi") Ikeda Shyoten. 2010.

7. Barkesgaard P, Heldt-Hansen HP, Diderichsen B. On the safety of Aspergillus oryzae: a review. Appl Microbiol Biotechnol. 1992;36:569-72.

8. Murakami H, Takase S, Ishii T. Non-productivity of aflatoxin by Japanese industrial strains of Aspergillus. I. Production of fluorescent substances in agar slant and shaking cultures. J Gen Appl Microbiol. 1967;13:323-34.

9. Tailor MJ, Richardson T. Application of microbial enzymes in food systems and in biotechnology. Adv Appl Microbiol. 1979;25:7-35.

10. Matsushima H, Chang P-K, Yu J, et al. Pre-termination in aflR of Aspergillus sojae inhibits aflatoxin biosynthesis. Appl Microbiol Biotechnol. 2001;55:585-9.

11. Tominaga M, Lee YH, Hayashi R, et al. Molecular analysis of an inactive aflatoxin biosynthesis gene claster in Aspergillus oryzae RIB strains. Appl Environ Microbiol. 2006;72:484-90.

12. Machida M, Asai K, Sano M, et al. Genome sequencing and analysis of Aspergillus oryzae. Nature. 2005;438:1157-61.

13. Sakaguchi K, Yamada K. Morphology and classification of Aspergillus fungi. Nihon Nogeikagaku Kaishi. J Agric Chem Soc Japan. 1944;20:65-73,141-4.

14. Sato A, Oshima K, Noguchi H, et al. Draft genome sequencing and comparative analysis of Aspergillus sojae NBRC4239. DNA Res. 2011a;18:165-76.

15. Inui $\mathrm{T}$. Ryukyu awamori hakko kin chyosa houkokusyo. J Tokyo Chem Soc. 1901a;22:669-88.
16. Inui $\mathrm{T}$. Untersuchungen über die niederen Organismen welche sich bei der Zubereitung des alkoholishen Getränkes "Awamori" betheiligen. J Coll Sci Imperial Univ Tokyo. 1901b;15:465-76.

17. Yang Y, Iwamoto A, Kumrungsa T, et al. Consumption of an acid protease derived from Aspergillus oryzae causes bifidogenic effect in rat. Nutrition Res. 2017;44:60-6.

18. Hong SB, Lee M, Kim DH, et al. Aspergillus luchuensis, an industrially important black Aspergillus in east Asia. PLoS ONE. 2013;8:e63769.

19. Hong SB, Yamada O, Samson RA. Taxonomic reevaluation of black koji mold. Appl Microbiol Biotechnol. 2014;98:555-61.

20. Ichishima E. Unique catalytic and molecular properties of hydrolases from Aspergillus used in Japanese bioindustries. Biosci Biotechnol Biochem. 2000;64:675-88.

21. Ichishima E. Unique enzymes of Aspergillus fungi used in Japanese bioindustries. Biochem Histochem Res. 2012;10:81-273.

22. Ichihshima E. Development of enzyme technology for Aspergillus oryzae, A. sojae, and A. luchuensis, the national microorganisms of Japan. Biosci Biotechnol Biochem. 2016;80:1681-92.

23. Brewing Society Japan. The national fungi of Japan. 2006.

24. Ichishima E. The national microorganism of Japan-Aspergilllus oryzae for society and culture. Tohoku University Press. 2017.

25. Ito K, Koyama Y, Hanya Y. Identification of the glutaminase gene of Aspergillus sojae involved in glutamate production during soy sauce fermentation. Biosci Biotechnol Biochem. 2013b;77:1832-40.

26. Ito $\mathrm{K}$, Matsushima $\mathrm{K}$, Koyama $\mathrm{Y}$. Gene cloning, purification, and characterization of a novel peptidoglutaninase-asparaginase from Aspergillus sojae. Appl Environ Microbiol. 2012;78:5182-8.

27. Ito K, Koyama Y. Analysis of specific digestion of the peptidoglutaminase-asparaginase of koji molds. J Biosci Bioeng. 2014;118:253-5.

28. Matsumoto I, Ohmoto M, Narukawa M, et al. Skn-1a/ Por2f3 specifies taste recettor cell lineage. Nat Neurosci. 2012; $14: 685-7$

29. Ushiama $\mathrm{S}$, Ishimaru $\mathrm{Y}$, Narukawa $\mathrm{M}$, et al. Catecholamine facilitate fuel expenditure and protect against via a network of the gut-brain axis in transcription factor Skn-1-deficient mice. EBioMedicine. 2016;8;60-71.

30. Maruyama J, Escaño CS, Kitamoto K. AoSO protein accumulated at the septal pore in response to various stresses in the filamentous fungus Aspergillus oryzae. Biochem Biophys Res Commun. 2010;391:868-73.

31. Han P, Jin FJ, Maruyama J, et al. A large nonconserved region of the tethering Protein Leashin is involved in regulating the position, movement, and function of Woronin bodies in Aspergillus oryzae. Eukaryot Cell. 2014;13:866-77.

32. Sato H, Toyoshima Y, Shintani T, et al. Identification of potential cell wall component that allows Taka-amylase an 
Citation: Eiji Ichishima. Bioscientific topics concerning Aspergillus oryzae, the national microorganism of Japan. J Biochem Biotech. 2018;1(1):42-46.

adsorption in submerged cultures of Aspergillus oryzae. Appl Microbiol Biotechnol. 2011b;92:961-6.

33. Ogawa M, Kobayashi T, Koyama Y. ManR, a novel $\mathrm{Zn}$ (II)2Cys6 transcriptional activator, controls the $\beta$ mannan utilization system in Aspergillus oryzae. Fungal Genet Biol. 2012;49:987-95.

34. Ogawa M, Kobayashi T, Koyama Y. ManR, a transcriptional regulator of the $\beta$-mannan utilization system, controls the cellulose utilization system in Aspergillus oryzae. Biosci Biotechnol Biochem. 2013;77;426-9.

35. Marui J, Matsushita-Morita M, Tada S, et al. Enzymatic properties of the glycine D-alanine aminopeptidase of Aspergillus oryzae and its activity profile in liquidcultured myceria and solid-state rice culture (rice koji). Appl Microbiol Biotechnol. 2012;93:655-69.

36. Takahashi $\mathrm{T}$, Maeda $\mathrm{H}$, Yoneda $\mathrm{S}$, et al. The fungal hydrophobin RolA recruits polyesterase and laterally moves on hydrophobic surfaces. Mol Microbiol. 2005;57:1780-96.

37. Maeda H, Yamagata Y, Abe K, et al. Purification and characterization of a biodegradable plastic-degrading enzyme from Aspergillus oryzae. Appl Microbiol Biotechnol. 2005;67:778-88.

38. Takahashi T, Tanaka T, Tsushima Y, et al. Ionic interaction of positive amino acid residues of fungal hydrophobin RolA with acidic amino acid residues of cutinase CutL1. Mol Microbiol. 2015;96:14-27.

39. Nakahara T, Yamaguchi H, Uchida R. Effect of temperature on the stability of various peptidases during peptide-enriched soy sauce fermentation. J Biosci Bioeng. 2012;113:353-9.

40. Ichishima E, Yoshida F. Molecular weight of acid proteinase of Aspergillus saitoi. Nature 1965;207:525-6

41. Gabeloteau C, Desnuelle P. On the activation of beef trypsinogen by a crystallized proteinase of Aspergillus saitoi. Biochim Biophys Acta. 1960;42:230-7.

42. Abita JP, Delaage M, Lazdunski M, et al. The mechanism of activation of trypsinogen. The role of the four Nterminal aspartyl residues. Eur J Biochem. 1969;8:314-24.

43. Shintani T, Ichishim E. Primary structure of aspergillopepsin I deduced from nucleotide sequence of the gene and aspartic acid-76 is an essential active site of the enzyme for trypsinogen activation. Biochim Biophys Acta. 1994;1204:257-64.

44. Shintani T, Kobayashi M, Ichishima E. Characterization of the S1 subsite specificity of aspergillopepsin I by site-directed mutagenesis. J Biochem. 1996;120:974-91.
45. Lee BR, Furukawa M, Yamashita K, et al. Aorsin, a novel serine proteinase with trypsin-like specificity at acidic $\mathrm{pH}$. Biochem J. 2003;371:541-8.

46. Ito K, Hanya Y, Kayama K. Purification and characterization of a glutaminase enzyme according for the majority of glutaminase in Aspergillus sojae under solid-state culture. Appl Microbiol Biotechnol. 2013a; 97:8581-90

47. Nakahara T, Sano A. Yamaguchi $M$, et al. Antihypertensive effect of peptide-enriched soy sauce-like seasoning and identification of its angiotensin-converting enzyme inhibitory substances. J Agric Food Chem. 2010;58:821-7.

48. Nakahara T, Sugimoto K, Sano A, et al. Antihypertensive mechanism of a peptide-enriched soy sauce-like seasoning: the active constituents and its suppressive effect on renin-angiotensin-aldosterone system. J Food Sci. 2011;76:201-6.

49. Nakanishi K. Trypsinogen-kinase in Aspergillus oryzae. III. Purification of trypsinogen kinase and its relation to acid-protease. J Biochem. 1959a;46:1263-9.

50. Nakanishi K. Trypsinogen-kinase in Aspergillus oryzae. IV. A study of acid-protease preparation. J Biochem. 1959b;46:1411-7.

51. Nakanishi K. Trypsinogen-kinase in Aspergillus oryzae. V. On the mechanism of activation. J Biochem. 1959c; 46:1553-8.

52. Udo S. Studies on the Umami taste of soy sauce. Correlation of the chemical components and the Umami taste of soy sauce. Nihon Nougeikagaku Kaishi. J Agric Chem Soc Japan. 1932;8:675-84.

53. Yamada O, Takara R, Hamada R, et al. Molecular biological researches of Kuro-Koji molds, their classification and safety. J Biosc Bioeng. 2011;112:233-7.

\section{*Corresponding author}

Eiji Ichishima

Department of Agricultural Chemistry,

Tokyo University of Agriculture and Technology,

3-15-17-101, Setagaya-ku, Tokyo,

Japan

Fax: 03-3326-6767

Tel: 03-3326-6767

E-Mail: ichie@cb3.so-net.ne.jp 